\title{
A Ramsey Treatment of Symmetry
}

\author{
$\begin{array}{lll}\text { T. Banakh* O. Verbitsky* }{ }^{\dagger} \quad \text { Ya. Vorobets } & \end{array}$ \\ Department of Mechanics and Mathematics \\ Lviv University, 79000 Lviv, Ukraine \\ E-mail: tbanakh@franko.lviv.ua
}

Submitted: November 8, 1999; Accepted: August 15, 2000.

But seldom is asymmetry merely the absence of symmetry. Hermann Weyl, "Symmetry"

\begin{abstract}
Given a space $\Omega$ endowed with symmetry, we define $m s(\Omega, r)$ to be the maximum of $m$ such that for any $r$-coloring of $\Omega$ there exists a monochromatic symmetric set of size at least $m$. We consider a wide range of spaces $\Omega$ including the discrete and continuous segments $\{1, \ldots, n\}$ and $[0,1]$ with central symmetry, geometric figures with the usual symmetries of Euclidean space, and Abelian groups with a natural notion of central symmetry. We observe that $m s(\{1, \ldots, n\}, r)$ and $m s([0,1], r)$ are closely related, prove lower and upper bounds for $m s([0,1], 2)$, and find asymptotics of $m s([0,1], r)$ for $r$ increasing. The exact value of $m s(\Omega, r)$ is determined for figures of revolution, regular polygons, and multi-dimensional parallelopipeds. We also discuss problems of a slightly different flavor and, in particular, prove that the minimal $r$ such that there exists an $r$-coloring of the $k$-dimensional integer grid without infinite monochromatic symmetric subsets is $k+1$.

MR Subject Number: 05D10

\footnotetext{
${ }^{*}$ Research supported in part by grant INTAS-96-0753.

${ }^{\dagger}$ Part of this work was done while visiting the Institute of Information Systems, Vienna University of Technology, supported by a Lise Meitner Fellowship of the Austrian Science Foundation (FWF).
} 
THE ELECTRONIC JOURNAL OF COMBINATORICS 7 (2000), \#R52

\section{$\S 0 \quad$ Introduction}

The aim of this work is, given a space with symmetry, to compute or to estimate the maximum size of a monochromatic symmetric set that exists for any $r$-coloring of the space.

More precisely, let $\Omega$ be a space with measure $\mu$. Suppose that $\Omega$ is endowed with a family $\mathcal{S}$ of transformations $s: \Omega \rightarrow \Omega$ called symmetries. A set $B \subseteq \Omega$ is symmetric if $s(B)=B$ for a symmetry $s \in \mathcal{S}$. An $r$-coloring of $\Omega$ is a map $\chi: \Omega \rightarrow\{1,2, \ldots, r\}$, where each color class $\chi^{-1}(i)$ for $i \leq r$ is assumed measurable. A set included into a color class is called monochromatic. In this framework, we address the value

$$
m s(\Omega, \mathcal{S}, r)=\inf _{\chi} \sup \{\mu(B): B \text { is a monochromatic symmetric subset of } \Omega\},
$$

where the infimum is taken over all $r$-colorings of $\Omega$. Our analysis covers the following spaces with symmetry.

$\S$ 1-2 Segments. $\mathcal{S}$ consists of central symmetries.

1 Discrete segment $\{1,2, \ldots, n\} \cdot \mu$ is the cardinality of a set.

2 Continuous segment $[0,1] \cdot \mu$ is the Lebesgue measure.

$\S 3$ Abelian groups. $\mathcal{S}$ consists of "central" symmetries $s_{g}(x)=g-x$.

3.1 Cyclic group $\mathbb{Z}_{n} \cdot \mu$ is the cardinality of a set. Equivalently: the vertex set of the regular $n$-gon with axial symmetry.

3.2 Group $\mathbb{R} / \mathbb{Z}$. $\mu$ is the Lebesgue measure. Equivalently: the circle with axial symmetry.

3.3 Arbitrary compact Abelian groups. $\mu$ is the Haar measure. A generalization of the preceding two cases.

$\S 4$ Geometric figures. $\mathcal{S}$ consists of non-identical isometries of $\Omega$ (including all central, axial, and rotational symmetries). $\mu$ is the Lebesgue measure.

4.1 Figures of revolution: disc, sphere etc.

4.2 Figures with finite $\mathcal{S}$ : regular polygons, ellipses and rectangles, their multidimensional analogs.

$\S 5$ analyses the cases when the value $m s(\Omega, \mathcal{S}, r)$ is attainable with a certain coloring $\chi$. $\S 6$ suggests another view of the subject with focusing on the cardinality of monochromatic symmetric subsets irrespective of the measure-theoretic aspects. $\S 7$ contains a list of open problems.

Techniques used for discrete spaces include a reduction to continuous optimization (Section 2.2), the probabilistic method (Proposition 2.6), elements of harmonic analysis (Proposition 3.4), an application of the Borsuk-Ulam antipodal theorem (Theorem 6.1). Continuous spaces are often approached by their discrete analogs (e.g. the segment and the circle are limit cases of the spaces $\{1,2, \ldots, n\}$ and $\mathbb{Z}_{n}$, respectively). In Section 4.1 combinatorial methods are combined with some Riemannian geometry and measure theory.

Throughout the paper $[n]=\{1,2, \ldots, n\}$. In addition to the standard $o$ - and $O$ notation, we write $\Omega(h(n))$ to refer to a function of $n$ that everywhere exceeds $c \cdot h(n)$, for 
THE ELECTRONIC JOURNAL OF COMBINATORICS 7 (2000), \#R52

$c$ a positive constant. The notation $\Theta(h(n))$ stands for a function that is simultaneously $O(h(n))$ and $\Omega(h(n))$. The relation $f(n) \sim h(n)$ means that $f(n)=h(n)(1+o(1))$.

All proofs that in this exposition are omitted or only sketched can be found in full detail in $[1,2,3,4,5,19,20,22]$ unless other sources are specified.

\section{$\S 1$ Discrete segment $[n]$}

\subsection{Warm-up}

A set $B \subseteq \mathbb{Z}$ such that $B=g-B$ for an integer $g$ is called symmetric (with respect to the center at rational point $\frac{1}{2} g$ ). Given a set of integers $A$, let $M S(A)$ denote the maximum cardinality of a symmetric subset $B \subseteq A$. In the case that $A \subseteq[n]$, notice the lower bound

$$
M S(A)>\frac{|A|^{2}}{2 n}
$$

Indeed, since there are $|A|^{2}$ ordered pairs $\left(a, a^{\prime}\right)$ of elements of $A$ and at most $2 n-1$ centers $\left(a+a^{\prime}\right) / 2$, at least $|A|^{2} /(2 n-1)$ pairs have a common center $g$.

Clearly, the maximum subset of $A$ symmetric with respect to $\frac{1}{2} g$ is $A \cap(g-A)$. The cardinality of $A \cap(g-A)$ is equal to the number of representations of $g$ as a sum $a+a^{\prime}$ with both $a$ and $a^{\prime}$ in $A$. This gives us some links to number theory.

Example 1.1 Primes - much symmetry.

Let $P_{\leq n}$ denote the set of all primes in $[n]$. The prime number theorem says that $\left|P_{\leq n}\right| \sim n / \log n$. It follows by (1) that $M S\left(P_{\leq n}\right)=\Omega\left(n / \log ^{2} n\right)$. This simple estimate turns out to be not so far from the true value $\Theta\left(\frac{n \log \log n}{\log ^{2} n}\right)$ due to Schnirelmann [21] and Prachar [18].

Example 1.2 Squares - little symmetry.

Let $S_{\leq n}$ denote the set of all squares in $[n]$. The Jacobi theorem says that if $g=2^{k} m$ with odd $m$, then the number of representations $g=x^{2}+y^{2}$ with integer $x$ and $y$ is equal to $4 E$, where $E$ denotes the excess of the number of divisors $t \equiv 1(\bmod 4)$ of $m$ over the number of its divisors $t \equiv 3(\bmod 4)$. The value $E$ does not exceed the number $d(m)$ of all positive divisors of $m$. It is known that $d(m)=m^{O(1 / \ln \ln m)}$ (Wigert, see also [16]). Therefore, $M S\left(S_{\leq n}\right)=n^{O(1 / \log \log n)}$.

Example 1.3 (Krückeberg [12]) A Sidon set - no symmetry.

Given a prime $p$, define the set $A_{p}=\left\{a_{1}, \ldots, a_{p}\right\}$ by $a_{i+1}=2 p i-\left(i^{2} \bmod p\right)+1$ for $0 \leq i<p$. This set turns out to be highly asymmetric, namely, $M S\left(A_{p}\right)=2$. Really, assume that $a_{i}+a_{j}=a_{i^{\prime}}+a_{j^{\prime}}$ with $i \leq j$ and $i^{\prime} \leq j^{\prime}$. From this it is easy to derive that

$$
\left\{\begin{array}{rlr}
i+j & =i^{\prime}+j^{\prime} & (\bmod p) \\
i^{2}+j^{2} & =\left(i^{\prime}\right)^{2}+\left(j^{\prime}\right)^{2} & (\bmod p)
\end{array}\right.
$$


Since in the field $\mathbb{F}_{p}$ a system of the kind

$$
\left\{\begin{array}{c}
i+j=a \\
i^{2}+j^{2}=b
\end{array}\right.
$$

can have only a unique solution $i, j$ with $i \leq j$, we conclude that $i=i^{\prime}$ and $j=j^{\prime}$, which proves the claim.

Sets $A$ with $M S(A)=2$, known as Sidon's sets or $B_{2}$-sequences, were investigated by many authors (see $[17$, section 4.1] for survey and references). For a Sidon set $A \subseteq[n]$ the estimate (1) implies $|A|<2 \sqrt{n}$. The stronger upper bound $|A| \leq \sqrt{n}(1+o(1))$ is due to Erdős and Turán. Thus, the set $A_{p}$ with the biggest $p \leq n$, for which $\left|A_{p}\right|=\sqrt{n}(1-o(1))$, is nearly as dense in $[n]$ as possible.

\subsection{Ramsey setting}

Given positive integers $n$ and $r$, consider the value

$$
M S(n, r)=\min _{\chi:[n] \rightarrow[r]} \max _{i \leq r} M S\left(\chi^{-1}(i)\right) .
$$

In other words, $M S(n, r)$ is the maximum integer such that for any $r$-coloring $\chi$ of $[n]$ there is a monochromatic symmetric subset $B \subseteq[n]$ with $|B| \geq M S(n, r)$.

For comparison let us define $M(n, r)$ in the same way with the only change that $B$ is now an arithmetic progression. Clearly, $M(n, r) \leq M S(n, r)$. In this notation the van der Waerden theorem (see $[11,15]$ ) says that $M(n, r) \rightarrow \infty$ as $n \rightarrow \infty$ for any fixed $r$, while the Berlekamp bound [6] reads to $M(n, r)=O(\log n)$. The function $M S(n, r)$ proves to grow much faster.

Proposition 1.4 For every $r$, the sequence $M S(n, r) / n$ converges as $n$ increases, and its limit is at least $1 /\left(2 r^{2}\right)$.

Proof. Observe relations

$$
\begin{aligned}
M S(k+j, r) & \leq M S(k, r)+2 j \\
M S(l \cdot n, r) & \leq l \cdot M S(n, r) .
\end{aligned}
$$

The first of them is obvious. To check the second, it suffices, given a coloring $\chi:[n] \rightarrow$ $[r]$, to consider the coloring $\chi^{\prime}:[\ln ] \rightarrow[r]$ such that $\chi^{\prime}(x)=\chi(\lceil x / l\rceil)$.

Let $j=m \bmod n$. By (3) and (4) we have

$$
\frac{M S(m, r)}{m} \leq \frac{M S(m-j, r)+2 j}{m} \leq \frac{M S(n, r)}{n}+\frac{2 j}{m} .
$$

Letting $m$ go to the infinity while keeping $n$ fixed, we obtain

$$
\limsup _{m \rightarrow \infty} \frac{M S(m, r)}{m} \leq \frac{M S(n, r)}{n} \text { for any } n \text {. }
$$

Hence the upper and lower limits of $M S(n, r) / n$ coincide, which implies the convergence. The estimate $\lim _{n \rightarrow \infty} M S(n, r) / n \geq 1 /\left(2 r^{2}\right)$ follows from (1). 
THE ELECTRONIC JOURNAL OF COMBINATORICS 7 (2000), \#R52

Notice that relation (5) has an important consequence.

Corollary $1.5 \lim _{n \rightarrow \infty} M S(n, r) / n$ exceeds no particular value $M S(n, r) / n$.

This fact suggests a way for computing upper bounds on $\lim _{n \rightarrow \infty} M S(n, r) / n$ as tight as desired. Unfortunately, computing $M S(n, r) / n$ seems not to be a feasible task for big $n$. Nonetheless, in Section 2.2 we achieve some speed-up in approaching the value $\lim _{n \rightarrow \infty} M S(n, r) / n$.

\subsection{General framework and the limit case of $[n]$}

The following definition gives the background for all further considerations. In particular, it will allow us to characterize the limit of $M S(n, r) / n$.

\section{Definition 1.6}

- Let $\mathcal{U}$ be a space with measure $\mu$.

- The space $\mathcal{U}$ is assumed to be endowed with a family $\mathcal{S}$ of one-to-one maps of $\mathcal{U}$ onto itself, that are measurable and preserve the measure. These maps will be called admissible symmetries.

- A set $B \subseteq \mathcal{U}$ is called symmetric if $s(B)=B$ for some symmetry $s \in \mathcal{S}$.

- Given $A \subseteq \mathcal{U}$, define

$$
m s(A)=\sup \{\mu(B): B \text { is a symmetric measurable subset of } A\} .
$$

- We consider a set $\Omega \subseteq \mathcal{U}$ with $\mu(\Omega)=1$, i.e. $(\Omega, \mu)$ is a probability space.

- Let $r \geq 2$. An $r$-coloring of $\Omega$ is a map $\chi: \Omega \rightarrow[r]$ such that each color class $\chi^{-1}(i)$ for $i \leq r$ is measurable. A subset of $\Omega$ is called monochromatic if it is included into a color class.

- Define

$$
m s(\Omega, r)=\inf _{\chi} \max _{i \leq r} m s\left(\chi^{-1}(i)\right),
$$

where the infimum is taken over all colorings of $\Omega$.

To avoid any ambiguity in the presence of several families of admissible symmetries, we will sometimes use more definite notation $m s(\Omega, \mathcal{S}, r)$. The notation $m s$ should be recognized as an abbreviation of "the maximal measure of a monochromatic symmetric subset". 
For example, consider $\Omega=[n]$ in $\mathcal{U}=\mathbb{Z}$. Let $\mu(x)=1 / n$ for every $x \in \mathcal{U}$. Let $\mathcal{S}$ consist of central symmetries $s(x)=g-x$ with center at point $g / 2$ for arbitrary integer $g$. Obviously, $m s([n], r)=M S(n, r) / n$.

Let $\Omega=[0,1]$ now be the unitary segment. Considering the universe $\mathcal{U}=\mathbb{R}$ with the Lebesgue measure and central symmetries with center at any real point, we obtain the definition of the value $m s([0,1], r)$. Proposition 1.4 can be made more precise.

Theorem $1.7 \lim _{n \rightarrow \infty} m s([n], r)=m s([0,1], r)$.

Estimation of $m s([0,1], r)$ will be our concern in the next section.

\section{$\S 2$ Continuous segment $[0,1]$}

In this section we estimate $m s([0,1], r)$ for $r=2$ and describe the asymptotic behavior of this value for $r \rightarrow \infty$.

\section{Theorem 2.1}

(1) $\frac{1}{4+\sqrt{6}} \leq m s([0,1], 2) \leq \frac{5}{24}$.

(2) $m s([0,1], r) \sim \frac{c}{r^{2}}$ for a constant $\frac{1}{2} \leq c \leq \frac{5}{6}$.

\subsection{Lower bound on $m s([0,1], 2)$}

We prove the lower bound in Theorem 2.1 (1) by the double-counting argument. Given $\epsilon>0$, fix a coloring of $[0,1]$ with color classes $A_{1}$ and $A_{2}$ such that both $m s\left(A_{i}\right)$ do not exceed $m s([0,1], 2)+\epsilon$. Consider Cartesian squares $A_{1}^{2}$ and $A_{2}^{2}$ in a plane. Obviously,

$$
\mu^{2}\left(A_{1}^{2} \cup A_{2}^{2}\right)=\mu\left(A_{1}\right)^{2}+\left(1-\mu\left(A_{1}\right)\right)^{2} \geq 1 / 2 .
$$

We now have to bound the left hand side of (6) from above. Define $S(a, b)=$ $\left\{(x, y) \in[0,1]^{2}: a \leq x+y \leq b\right\}$. Let $0<t<1$ be a parameter whose value will be chosen later. We split the square $[0,1]^{2}$ into three parts $S(0, t), S(t, 2-t)$, and $S(2-t, 2)$, and estimate the area of intersection of $A_{1}^{2} \cup A_{2}^{2}$ with each part separately.

Consider first the intersection with the strip $S(t, 2-t)$. From

$$
\begin{array}{r}
\mu\left(\left(A_{1}^{2} \cup A_{2}^{2}\right) \cap S(g, g)\right)=\sqrt{2}\left(\mu\left(A_{1} \cap\left(g-A_{1}\right)\right)+\mu\left(A_{2} \cap\left(g-A_{2}\right)\right)\right) \\
\leq 2 \sqrt{2}(m s([0,1], 2)+\epsilon)
\end{array}
$$

we infer that

$$
\mu^{2}\left(\left(A_{1}^{2} \cup A_{2}^{2}\right) \cap S(t, 2-t)\right) \leq 4(1-t)(m s([0,1], r)+\epsilon) .
$$

To estimate the intersection with the triangle $S(0, t)$, we use two lemmas. 
Lemma 2.2 If $B \subseteq[0, t]$, then $\mu(B) \leq(t+m s(B)) / 2$.

Proof. Consider the partition of $B$ into three parts $B^{\prime}=B \cap(t-B), B^{\prime \prime}=(B \backslash$ $\left.B^{\prime}\right) \cap[0, t / 2]$, and $B^{\prime \prime \prime}=\left(B \backslash B^{\prime}\right) \cap[t / 2, t]$. Since sets $B^{\prime} \cap[0, t / 2], B^{\prime \prime}$, and $t-B^{\prime \prime \prime}$ do not intersect, we have $\mu\left(B^{\prime}\right) / 2+\mu\left(B^{\prime \prime}\right)+\mu\left(B^{\prime \prime \prime}\right) \leq t / 2$. As $\mu\left(B^{\prime}\right) \leq m s(B)$, we obtain $\mu(B)=\mu\left(B^{\prime}\right) / 2+\mu\left(B^{\prime}\right) / 2+\mu\left(B^{\prime \prime}\right)+\mu\left(B^{\prime \prime \prime}\right) \leq m s(B) / 2+t / 2$.

For $B_{i}=A_{i} \cap[0, t]$, Lemma 2.2 implies that $\mu\left(B_{i}\right) \leq(t+m s([0,1], 2)+\epsilon) / 2$.

Lemma 2.3 Given a partition $[0, t]=B_{1} \cup B_{2}$, suppose that $\max \left\{\mu\left(B_{1}\right), \mu\left(B_{2}\right)\right\} \leq s$, where

$$
s \geq \frac{2}{3} t
$$

Then

$$
\mu\left(\left(B_{1}^{2} \cup B_{2}^{2}\right) \cap S(0, t)\right) \leq s^{2}-(2 s-t) / 2 .
$$

An equivalent reformulation of the lemma is that the area of $\left(B_{1}^{2} \cup B_{2}^{2}\right) \cap S(0, t)$ attains its maximum at the partition $B_{1}=[0, s], B_{2}=[s, t]$. This fact is not so obvious as it appears at first sight, say, it is not true if the condition (8) is violated. The proof is omitted in this exposition (see [5, lemma 6.12] for details).

Assuming that $m s([0,1], 2)<1 / 3$ (otherwise nothing to prove), we set

$$
t=3 m s([0,1], 2) .
$$

Apply Lemma 2.3 to the partition of $[0, t]$ into $B_{i}=A_{i} \cap[0, t], i=1,2$, with $s=$ $(t+m s([0,1], 2)+\epsilon) / 2=2 m s([0,1], 2)+\epsilon / 2$. As the condition (8) is fulfilled, we obtain

$$
\mu^{2}\left(\left(A_{1}^{2} \cup A_{2}^{2}\right) \cap S(0, t)\right)=\mu^{2}\left(\left(B_{1}^{2} \cup B_{2}^{2}\right) \cap S(0, t)\right) \leq \frac{7}{2} m s([0,1], 2)^{2}+O(\epsilon) .
$$

The same bound holds true for the intersection $\left(A_{1}^{2} \cup A_{2}^{2}\right) \cap S(2-t, 2)$. Summing it up with (9) and (7), we obtain an upper bound on $\mu^{2}\left(A_{1}^{2} \cup A_{2}^{2}\right)$ which after comparison with the lower bound (6) implies

$$
10 m s([0,1], 2)^{2}-8 m s([0,1], 2)+1 \leq O(\epsilon) .
$$

As $\epsilon$ can be here arbitrarily small, the bound $m s([0,1], 2) \geq 1 /(4+\sqrt{6})$ follows.

\subsection{Blurred colorings}

For the remaining claims of Theorem 2.1 we need to involve some machinery. The idea is to move from our problem to its (hopefully) more tractable continuous version. For this purpose we modify the notion of coloring, allowing a point $x \in \Omega$ be colored by several colors mixed in arbitrary proportion. The fraction of each color at $x$ is a non-negative real number, and the sum of all color fractions should equal 1. A similar concept of the fractional coloring of a graph is well known in combinatorics and discrete optimization. However our approach is different in some important aspects; in particular, our problem seems to fall out from the scope of linear or even convex programming. This justifies our choice of other term blurred coloring. 
THE ELECTRONIC JOURNAL OF COMBINATORICS 7 (2000), \#R52

\section{Definition 2.4}

- Let a space $\mathcal{U}$ with measure $\mu$, a set $\Omega \subseteq \mathcal{U}$, and a family of symmetries $\mathcal{S}$ satisfy the conditions of Definition 1.6. Assume in addition that every symmetry $s \in \mathcal{S}$ is involutive, i.e. $s=s^{-1}$.

- A blurred $r$-coloring of $\Omega$ is an arbitrary set of measurable functions $\left\{\beta_{i}: \mathcal{U} \rightarrow\right.$ $[0,1]\}_{i=1}^{r}$ such that $\sum_{i=1}^{r} \beta_{i}=\chi_{\Omega}$, where $\chi_{\Omega}$ denotes the characteristic function of $\Omega$.

- Given a measurable function $f: \mathcal{U} \rightarrow \mathbb{R}$, we define a map $f \star f: \mathcal{S} \rightarrow \mathbb{R}$ by

$$
f \star f(s)=\int_{\mathcal{U}} f(x) f(s(x)) d \mu(x) .
$$

We use the notation $\|\cdot\|$ for the uniform norm on the set of functions from $\mathcal{S}$ to $\mathbb{R}$, i.e. $\|F\|=\sup _{s \in \mathcal{S}}|F(s)|$ for a function $F: \mathcal{S} \rightarrow \mathbb{R}$.

- An analog of the maximum measure of a monochromatic symmetric subset under a blurred coloring $\beta=\left\{\beta_{i}\right\}_{i=1}^{r}$ is defined by

$$
\operatorname{bms}(\Omega, r ; \beta)=\max _{i \leq r}\left\|\beta_{i} \star \beta_{i}\right\| .
$$

We set

$$
b m s(\Omega, r)=\inf _{\beta} b m s(\Omega, r ; \beta),
$$

where the infimum is taken over all blurred $r$-colorings of $\Omega$.

Proposition 2.5 For every space $\Omega$ with involutive symmetries we have bms $(\Omega, r) \leq$ $m s(\Omega, r)$.

Proof-sketch. It suffices to observe that the notion of a blurred coloring generalizes the notion of a coloring that has been considered so far. An ordinary "distinct" coloring $\chi$ of $\Omega$ can be viewed as a blurred coloring $\beta=\left\{\beta_{i}: \mathcal{U} \rightarrow[0,1]\right\}_{i=1}^{r}$ taking on only two values 0 and 1 in the segment $[0,1]$ so that $\beta_{i}(x)=1$ whenever $\chi(x)=i$ and $\beta_{i}(x)=0$ otherwise.

In a rather typical situation the values $m s(\Omega, r)$ and $b m s(\Omega, r)$ turn out to be close to each other. To be more precise, suppose that $\Omega$ is a finite subset of the universe $\mathcal{U}$, every finite set $A \subseteq \mathcal{U}$ has measure $\mu(A)=|A| /|\Omega|$, and the family of symmetries $\mathcal{S}$ consists of involutions. Given a symmetry $s \in \mathcal{S}$, let $\operatorname{Fix}(s)=\{x \in \Omega: s(x)=x\}$.

Proposition 2.6 Let $n=|\Omega|$ and $m=\max _{s \in \mathcal{S}}|\operatorname{Fix}(s)|$. Then

$$
m s(\Omega, r) \leq b m s(\Omega, r)+\frac{m}{n}+\left(\frac{4 \ln (r|\mathcal{S}|)}{n-m}\right)^{1 / 2}
$$


THE ELECTRONIC JOURNAL OF COMBINATORICS 7 (2000), \#R52

Proof-sketch. $\quad$ Since $\Omega$ is finite, $b m s(\Omega, r)=b m s(\Omega, r ; \beta)$ for some blurred coloring $\beta=\left\{\beta_{i}\right\}_{i=1}^{r}$. Define a random distinct coloring $\chi$ so that each point $x \in \Omega$ receives color $i$ with probability $\beta_{i}(x)$, independently of other points. With nonzero probability, every $\chi$-monochromatic symmetric subset of $\Omega$ has measure no more than the right hand side of $(10)$.

\subsection{Upper bound on $m s([0,1], 2)$}

Recall that by Corollary 1.5 the values $m s([n], r)$ approximate $m s([0,1], r)$ from above. Let us show that the values $b m s([n], r)$ do the same as well (and likely even better).

Applying Propositions 2.5 and 2.6 to the discrete space $[n]$, we obtain

$$
b m s([n], r) \leq m s([n], r) \leq b m s([n], r)+o(1)
$$

for a fixed $r$ and $n$ increasing. By Theorem 1.7 this implies that

$$
b m s([n], r) \rightarrow m s([0,1], r) \text { as } n \rightarrow \infty
$$

Similarly to relations (3) and (4), one can prove their counterparts

$$
\begin{aligned}
b m s([k+j], r) & \leq b m s([k], r) \frac{k}{k+j}+\frac{2 j}{k+j} \\
b m s([l n], r) & \leq b m s([n], r) .
\end{aligned}
$$

In the same vein as in Section 1.2, we derive from here that

$$
\lim _{m \rightarrow \infty} b m s([m], r) \leq b m s([n], r)
$$

for all $n$. By (12) we get

$$
m s([0,1], r) \leq b m s([n], r)
$$

for all $n$. We gain from (13) even with small $n$. To prove the desired bound $m s([0,1], r) \leq$ $5 / 24$ we just set $n=4$ and apply the following fact.

Lemma $2.7 \operatorname{bms}([4], 2) \leq 5 / 24$.

Proof. Consider the blurred coloring $\beta=\left\{\beta_{1}, 1-\beta_{1}\right\}$ with

$$
\beta_{1}(1)=\frac{1}{2}, \beta_{1}(2)=\frac{1}{2}-\frac{1}{2 \sqrt{3}}, \beta_{1}(3)=\frac{1}{2}+\frac{1}{2 \sqrt{3}}, \beta_{1}(4)=\frac{1}{2} .
$$

Straightforward computation shows that $\operatorname{bms}([4], 2 ; \beta)=5 / 24$. 
THE ELECTRONIC JOURNAL OF COMBINATORICS 7 (2000), \#R52

\subsection{Asymptotics of $m s([0,1], r)$ for $r \rightarrow \infty$}

In this section we prove the second statement of Theorem 2.1. We again prefer to deal with blurred colorings. In the case of the segment this is reasonable because

$$
b m s([0,1], r)=m s([0,1], r)
$$

This equality is true because, simultaneously with $(12), \operatorname{bms}([n], r) \rightarrow \operatorname{bms}([0,1], r)$ as $n \rightarrow \infty$. The latter convergence is an analog of Theorem 1.7 and is provable by essentially the same argument (see [5] for details).

Our next goal is to check the inequality

$$
\limsup _{r \rightarrow \infty} b m s([0,1], r) r^{2} \leq b m s([0,1], k) k^{2}
$$

for any fixed $k$. Given $\epsilon>0$, let $\beta=\left\{\beta_{i}\right\}_{i=0}^{k-1}$ be a blurred $k$-coloring of $[0,1]$ with $b m s([0,1], k ; \beta)<b m s([0,1], k)+\epsilon$. Assume $r=k t$ and define a blurred $r$-coloring $\chi=\left\{\chi_{i}\right\}_{i=0}^{r-1}$ by $\chi_{i}(x)=\frac{1}{t} \beta_{i \bmod k}(x)$ for all $x \in[0,1]$. Then

$$
\begin{array}{r}
\operatorname{bms}([0,1], r) \leq b m s([0,1], r ; \chi)=\max _{0 \leq i<r}\left\|\chi_{i} \star \chi_{i}\right\|= \\
\max _{0 \leq i<k} \frac{1}{t^{2}}\left\|\beta_{i} \star \beta_{i}\right\|=\frac{1}{t^{2}} b m s([0,1], k ; \beta)<\frac{1}{t^{2}} b m s([0,1], k)+\epsilon .
\end{array}
$$

As $\epsilon$ can be arbitrarily small, we obtain relation $b m s([0,1], r) \leq \frac{k^{2}}{r^{2}} b m s([0,1], k)$ for $r$ multiple of $k$. For arbitrary $r$, letting $j=r \bmod k$ we obtain

$$
b m s([0,1], r) \leq b m s([0,1], r-j) \leq \frac{k^{2}}{r^{2}} b m s([0,1], k)\left(\frac{r}{r-j}\right)^{2}
$$

and inequality (15) follows.

From (15) we conclude that the upper and lower limits of $b m s([0,1], r) r^{2}$ for $r \rightarrow \infty$ coincide, and hence there exists $\lim _{r \rightarrow \infty} b m s([0,1], r) r^{2}=c$. By equality (14) we obtain $m s([0,1], r) \sim \frac{c}{r^{2}}$.

The bound $c \geq 1 / 2$ follows from the relation $m s([0,1], r) \geq 1 /\left(2 r^{2}\right)$ (see Proposition 1.4 and Theorem 1.7). To prove the bound $c \leq 5 / 6$ it suffices to put $k=2$ in (15) and use inequalities bms $([0,1], 2) \leq b m s([4], 2) \leq 5 / 24$.

\section{$\S 3$ Abelian groups}

The notion of symmetry in $\mathbb{Z}$ or $\mathbb{R}$ can be naturally extended to any Abelian group. More precisely, two families of symmetries look reasonable for an Abelian group $G$.

$\mathcal{S}$ - the family of "central" symmetries $s: G \rightarrow G$ of kind $s(x)=2 g-x$ for some $g \in G$ 
THE ELECTRONIC JOURNAL OF COMBINATORICS 7 (2000), \#R52

$\mathcal{S}_{+}-$the extended family of symmetries $s: G \rightarrow G$ of kind $s(x)=g-x$ for some $g \in G$.

Given a finite group $G$, we consider the counting measure $\mu$, i.e. $\mu(A)=|A| /|G|$ for any $A \subseteq G$. Given the group $\mathbb{R} / \mathbb{Z}$, which can be viewed equivalently as the unitary circle in the complex plane, we consider the Lebesgue measure. Both cases are covered by the most general setting where we consider the Haar measure on a compact Abelian group $G$.

Therewith every compact Abelian group $G$ can be regarded as a space with symmetry. To shorten notation, we set $m s(G, r)=m s(G, \mathcal{S}, r)$ and $m s_{+}(G, r)=m s\left(G, \mathcal{S}_{+}, r\right)$. As $\mathcal{S} \subseteq \mathcal{S}_{+}$, it holds $m s(G, r) \leq m s_{+}(G, r)$.

\subsection{Cyclic group $\mathbb{Z}_{n}$}

Consideration of $\mathbb{Z}_{n}$ has a distinct geometric sense, since $\mathbb{Z}_{n}$ can be viewed as the vertex set of the rectangular $n$-gon. Then $\mathcal{S}$ consists of reflections in those axes that pass through a vertex, while $\mathcal{S}_{+}$consists of all axial symmetries. Another reason why $\mathbb{Z}_{n}$ deserves a detailed treatment is that this is the model case for a wide variety of compact Abelian groups.

Notice that if $n$ is odd, then $\mathcal{S}=\mathcal{S}_{+}$and hence $m s\left(\mathbb{Z}_{n}, r\right)=m s_{+}\left(\mathbb{Z}_{n}, r\right)$. In this section we prove the following result.

Theorem 3.1 For a fixed number of colors $r$ and $n$ increasing we have

$$
1 / r^{2} \leq m s\left(\mathbb{Z}_{n}, r\right) \leq m s_{+}\left(\mathbb{Z}_{n}, r\right) \leq 1 / r^{2}+o(1)
$$

Moreover, it holds the strict inequality

$$
m s_{+}\left(\mathbb{Z}_{n}, r\right)>1 / r^{2}
$$

Lower bounds. Recall that $\mu(A)=|A| / n$ is the density of a set $A \subseteq \mathbb{Z}_{n}$. Let $\chi_{A}: \mathbb{Z}_{n} \rightarrow\{0,1\}$ denote the characteristic function of $A$. Define

$$
f(g)=\mu(A \cap(g-A))=\frac{1}{n} \sum_{x \in \mathbb{Z}_{n}} \chi_{A}(x) \chi_{A}(g-x)
$$

to be the density of the maximum subset of $A$ symmetric with respect to symmetry $s(x)=g-x$. The proof of lower bounds in Theorem 3.1 is based on the simple observation that at least one of $r$ color classes must have density at least $1 / r$. The weakest bound $m s_{+}\left(\mathbb{Z}_{n}, r\right) \geq 1 / r^{2}$ immediately follows from the statement below.

Proposition 3.2 Every set $A \subseteq \mathbb{Z}_{n}$ contains an $\mathcal{S}_{+}$-symmetric subset of density at least $\mu(A)^{2}$. 
THE ELECTRONIC JOURNAL OF COMBINATORICS 7 (2000), \#R52

Proof. We apply the standard averaging argument. Using (18), we have

$$
\frac{1}{n} \sum_{g \in \mathbb{Z}_{n}} f(g)=\frac{1}{n} \sum_{x \in \mathbb{Z}_{n}} \chi_{A}(x) \frac{1}{n} \sum_{g \in \mathbb{Z}_{n}} \chi_{A}(g-x)=\mu(A)^{2} .
$$

Therefore, $f(g) \geq \mu(A)^{2}$ for at least one $g$.

The next two statements strengthen Proposition 3.2 in two different directions. The first of them implies the bound $m s\left(\mathbb{Z}_{n}, r\right) \geq 1 / r^{2}$ in Theorem 3.1.

Proposition 3.3 Every set $A \subseteq \mathbb{Z}_{n}$ contains an $\mathcal{S}$-symmetric subset of density at least $\mu(A)^{2}$.

Proof. For odd $n$ the statement coincides with Proposition 3.2. Suppose that $n=2 m$. Let $A_{0}$ and $A_{1}$ be two parts of $A$ consisting of even and odd numbers respectively. Averaging (18) on even arguments of $f$, we obtain

$$
\begin{array}{r}
\frac{1}{m} \sum_{g \in \mathbb{Z}_{m}} f(2 g)=\frac{1}{n} \sum_{x \in \mathbb{Z}_{n}} \chi_{A}(x) \frac{1}{m} \sum_{g \in \mathbb{Z}_{m}} \chi_{A}(2 g-x)= \\
\frac{1}{n} \sum_{x \text { even }} \chi_{A_{0}}(x) \frac{1}{m} \sum_{x \text { even }} \chi_{A_{0}}(x)+\frac{1}{n} \sum_{x \text { odd }} \chi_{A_{1}}(x) \frac{1}{m} \sum_{x \text { odd }} \chi_{A_{1}}(x)= \\
2 \mu\left(A_{0}\right)^{2}+2 \mu\left(A_{1}\right)^{2} \geq\left(\mu\left(A_{0}\right)+\mu\left(A_{1}\right)\right)^{2}=\mu(A)^{2} .
\end{array}
$$

Therefore, $f(2 g) \geq \mu(A)^{2}$ for at least one $g$.

It remains to prove the bound $m s_{+}\left(\mathbb{Z}_{n}, r\right)>1 / r^{2}$ in Theorem 3.1.

Proposition 3.4 Let $A$ be a proper nonempty subset of $\mathbb{Z}_{n}$. Then $A$ contains an $\mathcal{S}_{+}$symmetric subset of density strictly more than $\mu(A)^{2}$.

Proof. Assume, to the contrary, that $f(g) \leq \mu(A)^{2}$ for all $g$. By (19) this implies $f(g) \equiv \mu(A)^{2}$, where $\equiv$ means equality everywhere on $\mathbb{Z}_{n}$.

Let $\phi_{i}: \mathbb{Z}_{n} \rightarrow \mathbb{C}$, for $0 \leq i<n$, be all characters of $\mathbb{Z}_{n}$, that is, all homomorphisms from $\mathbb{Z}_{n}$ to $\mathbb{C}$. The system $\left\{\phi_{i}\right\}_{i=0}^{n-1}$ is an orthonormal basis of the Hilbert space $L_{2}\left(\mathbb{Z}_{n}\right)=$ $\mathbb{C}^{\mathbb{Z}_{n}} \simeq \mathbb{C}^{n}$. This is a general property of characters of a compact Abelian group (see e.g. $[13, \S 38])$, which in the case of $\mathbb{Z}_{n}$ reduces to the non-singularity of the Vandermonde matrix. We will suppose that $\phi_{0} \equiv 1$.

Relation (18) shows that the function $f$ is representable as the convolution $\chi_{A} \star \chi_{A}$. Assuming the expansion $\chi_{A}=\sum_{i=0}^{n-1} c_{i} \phi_{i}$ in the basis $\left\{\phi_{i}\right\}_{i=0}^{n-1}$, we obtain $f=\sum_{i=0}^{n-1} c_{i}^{2} \phi_{i}$. Comparing this with $f \equiv \mu(A)^{2}$, from the uniqueness of expansion we conclude that $c_{0}^{2}=\mu(A)^{2}$, while all the other coefficients $c_{i}$ are zero. Thus, $\chi_{A} \equiv \mu(A)$ and $A$ must be either $\emptyset$ or $\mathbb{Z}_{n}$, a contradiction. 
THE ELECTRONIC JOURNAL OF COMBINATORICS 7 (2000), \#R52

The upper bound in Theorem 3.1 is a direct consequence of Proposition 2.6 that in the case $\Omega=\mathbb{Z}_{n}$ reads as follows.

Proposition $3.5 m s_{+}\left(\mathbb{Z}_{n}, r\right) \leq 1 / r^{2}+O(\sqrt{\log (r n) / n})$.

Indeed, in notation of Proposition 2.6 we have $m \leq 2$. Moreover, for any space $\Omega$ we have $b m s(\Omega, r) \leq 1 / r^{2}$ as follows from consideration of the blurred coloring $\left\{\beta_{i}\right\}_{i=1}^{r}$ with each $\beta_{i}=1 / r$ everywhere on $\Omega$.

\subsection{Circle $\mathbb{R} / \mathbb{Z}$}

The group $\mathbb{R} / \mathbb{Z}$ is of especial interest because it can be alternatively viewed as the circle with axial symmetry. Of course, $\mathcal{S}=\mathcal{S}_{+}$.

Theorem 3.6 ms $(\mathbb{R} / \mathbb{Z}, r)=1 / r^{2}$.

The proof of Theorem 3.6 borrows much from our analysis of $\mathbb{Z}_{n}$. Similarly to $\mathbb{Z}_{n}$, the following properties are true for $\Omega=\mathbb{R} / \mathbb{Z}$.

(L) Every measurable set $A \subseteq \Omega$ contains a symmetric subset $B \subseteq A$ of measure $\mu(B) \geq \mu(A)^{2}$.

(SL) Every measurable set $A \subset \Omega$ of measure $0<\mu(A)<1$ contains a symmetric subset $B \subseteq A$ of measure $\mu(B)>\mu(A)^{2}$.

(U) $m s(\Omega, r) \leq 1 / r^{2}$.

The proof of $(\mathrm{L})$ is the same as that of Proposition 3.2, with integration instead of summation. As a consequence, $m s(\mathbb{R} / \mathbb{Z}, r) \geq 1 / r^{2}$. Property (SL), the remarkable strengthening of $(\mathrm{L})$, can be proved with using the Fourier expansion similarly to Proposition 3.4. Property (U) is provable by reduction to Proposition 3.5 on account of the following fact.

Proposition 3.7 Let $H$ be a finite subgroup of a compact Abelian group $G$. Then $m s_{+}(G, r) \leq m s_{+}(H, r)$.

We therefore have $m s(\mathbb{R} / \mathbb{Z}, r) \leq m s_{+}\left(\mathbb{Z}_{n}, r\right) \leq \frac{1}{r^{2}}+O(\sqrt{\log (r n) / n})$ for all $n$, which immediately implies (U).

We will refer to Properties (L), (SL), and (U) in the rest of the survey as they are common for many spaces with symmetry. 
THE ELECTRONIC JOURNAL OF COMBINATORICS 7 (2000), \#R52

\subsection{Arbitrary compact Abelian groups}

Recall that we consider a compact Abelian group $G$ along with its Haar measure $\mu$. The topology of $G$ is assumed Hausdorff, and $\mu$ is assumed to be a complete probability measure. This setting includes the groups $\mathbb{Z}_{n}$ with the counting measure and $\mathbb{R} / \mathbb{Z}$ with the Lebesgue measure as particular cases.

Theorem 3.8 Let $[G]_{2}$ denote the subgroup of a group $G$ consisting of the elements of order 2. Then $m s(G, r)=m s_{+}(G, r)=1 / r^{2}$ provided $\mu\left([G]_{2}\right)=0$.

The lower bound $m s(G, r) \geq 1 / r^{2}$ follows from Property (L) above that is true for every compact Abelian group $\Omega=G$ with respect to the family of symmetries $\mathcal{S}$. Moreover, Property (SL) is true with respect to the extended family of symmetries $\mathcal{S}_{+}$. To establish Property $(\mathrm{U})$ with respect to $\mathcal{S}_{+}$, the following relation is useful.

Proposition 3.9 Let $H$ be a closed subgroup of a compact Abelian group $G$. Then $m s_{+}(G, r) \leq m s_{+}(G / H, r)$.

Proving (U), we distinguish two cases. If there exists a homomorphism from $G$ onto $\mathbb{R} / \mathbb{Z}$, then (U) follows from Proposition 3.9 and Theorem 3.6. Otherwise, the structural theory of compact Abelian groups (see e.g. [14]) implies that $G$ can be approximated by a sequence of finite Abelian groups $\left\{D_{n}\right\}$ in the sense that $G$ has closed subgroups $H_{n}$ with $G / H_{n} \simeq D_{n}$ and $\mu\left(H_{n}\right) \rightarrow 0$. By Proposition 3.9, $m s_{+}(G, r) \leq m s_{+}\left(D_{n}, r\right)$. It remains to prove the upper bound $m s_{+}\left(D_{n}, r\right)=1 / r^{2}+o(1)$, what can be done by the probabilistic method similarly to Proposition 3.5. As an example of this scenario one can suggest the group $Z(p)$ of integer $p$-adic numbers, which is approximated in the above sense by the cyclic groups $\mathbb{Z}_{p^{n}}$.

\section{$\S 4$ Geometric figures}

This section is devoted to symmetric geometric figures in Euclidean space $\mathbb{R}^{k}$. The general reference books on the topic are [8, 23]. We consider two classes of figures that require completely different approaches. One class consists of surfaces and bodies of revolution. Another class includes plane figures like regular polygons, ellipses and rectangles (equivalent as spaces with symmetry), and their multi-dimensional analogs. The crucial feature of this class is that its members have only finitely many symmetries.

Every figure $\Omega$ is considered with the Lebesgue measure $\mu$ on $\Omega$ normed so that $\mu(\Omega)=1$. The family of admissible symmetries consists of all non-identical isometries of $\mathbb{R}^{k}$ leaving $\Omega$ invariant. We therewith have defined the value $m s(\Omega, r)$. 
THE ELECTRONIC JOURNAL OF COMBINATORICS 7 (2000), \#R52

\subsection{Figures of revolution}

Though our results apply to a wide range of figures of revolution including cylinder, cone, torus etc., we will focus on the ball $V^{k}$ and the sphere $S^{k-1}$ in Euclidean space of dimension $k$. We adopt formulations of Properties (L), (SL), and (U) from Section 3.2.

\section{Theorem 4.1}

(1) The spaces $\Omega=S^{k-1}$ and $\Omega=V^{k}$ for any $k \geq 2$ have Properties (L) and $(U)$. Consequently, $m s(\Omega, r)=1 / r^{2}$.

(2) The sphere $S^{k}$ for $k \geq 1$ and the ball $V^{k}$ for $k \geq 3$ have Property (SL).

(3) The disc $V^{2}$ does not have Property (SL). Moreover, there is an $r$-coloring of $V^{2}$ without monochromatic symmetric subsets of measure more than $1 / r^{2}$.

Theorem 4.1 strengthens Property (U) shown in Section 3.2 for the circle $S^{1}$, as now this property is stated not only for bilateral but also for rotatory symmetry. In general, Theorem 4.1 states the upper bounds (i.e. Property (U) and negation of (SL)) for the fairly rich family of all non-identical isometries of a figure. On the other hand, the lower bounds (L) and (SL) will be actually proved for much more limited family of symmetries consisting of reflections in hyperplanes. This makes our results stronger, as decrease of admissible symmetries can make the value $m s(A)$ for $A \subseteq \Omega$ only smaller.

Property (L) follows from the argument common for all figures of revolution. From the measure-theoretic point of view any figure of revolution $\Omega$ is representable as the product $\Omega=S^{1} \times \Omega_{1}$ of the circle and some probability space $\Omega_{1}$. Correspondingly, $\Omega$ has the product-measure $\mu=\mu_{0} \times \mu_{1}$, where $\mu_{0}$ denotes the probability Lebesgue measure on $S^{1}$, and $\mu_{1}$ is the measure on $\Omega_{1}$. Identifying the circle $S^{1}$ with the group $\mathbb{R} / \mathbb{Z}$, for each $g \in S^{1}$ we consider symmetry $s_{g}\left(x, x_{1}\right)=\left(g-x, x_{1}\right)$, where $x \in S^{1}$ and $x_{1} \in \Omega_{1}$. Notice that any such symmetry is reflection in a hyperplane.

Proposition 4.2 Every measurable set $A \subseteq S^{1} \times \Omega_{1}$ contains a symmetric subset $B \subseteq A$ of measure $\mu(B) \geq \mu(A)^{2}$.

Proof. Let $B_{g}=A \cap s_{g}(A)$ be the maximum subset of $A$ symmetric with respect to a symmetry $s_{g}$. Denote $A_{x_{1}}=\left\{x \in S^{1}:\left(x, x_{1}\right) \in A\right\}$, a section of the set $A$. Representing $\mu\left(B_{g}\right)$ as the integral of the characteristic function of the set $B_{g}$, averaging it on $g$ and changing the order of integration, we come to the equality $\int_{S^{1}} \mu\left(B_{g}\right) d \mu_{0}(g)=$ $\int_{\Omega_{1}} \mu_{0}\left(A_{x_{1}}\right)^{2} d \mu_{1}\left(x_{1}\right)$. Applying the Cauchy-Schwartz inequality, we obtain

$$
\int_{S^{1}} \mu\left(B_{g}\right) d \mu_{0}(g)=\int_{\Omega_{1}} \mu_{0}\left(A_{x_{1}}\right)^{2} d \mu_{1}\left(x_{1}\right) \geq\left(\int_{\Omega_{1}} \mu_{0}\left(A_{x_{1}}\right) d \mu_{1}\left(x_{1}\right)\right)^{2}=\mu(A)^{2} .
$$

There must exist $g \in S^{1}$ such that $\mu\left(B_{g}\right) \geq \mu(A)^{2}$. 
Property (U). In fact, we are able to prove the bound $m s(\Omega, r) \leq 1 / r^{2}$ in a very general form, namely, for $\Omega$ being any compact subset of a connected Riemannian manifold. The basic idea is the same as in the proof of Proposition 3.5 where we, in essence, show that large monochromatic symmetric subsets in $\mathbb{Z}_{n}$ are avoidable by coloring $\mathbb{Z}_{n}$ at random. In a similar vein, we partition $\Omega$ into small measurable pieces and color it piecewise at random. Then we show that with nonzero probability there is no monochromatic symmetric set whose measure exceeds $1 / r^{2}+\epsilon$, for a small $\epsilon>0$. The obvious bottleneck in this scenario is that most often the family $\mathcal{S}$ of symmetries is infinite. Nonetheless, we manage to approximate $\mathcal{S}$ by its finite subset in the metric $\rho\left(s_{1}, s_{2}\right)=\sup _{x \in \Omega} \operatorname{dist}\left(s_{1}(x), s_{2}(x)\right)$, where dist denotes the distance between two points in $\mathbb{R}^{k}$. The complete proof contains some subtleties and is given in [5].

Property (SL) was already stated in Section 3.2 for the circle $S^{1}$. For spheres and balls in higher dimensions we use a different argument. To facilitate the exposition, we prove the claim 2 of Theorem 4.1 only for the sphere $S^{2}$.

Proposition 4.3 Every subset $A \subset S^{2}$ of measure $0<\mu(A)<1$ contains a symmetric subset $B$ of measure $\mu(B)>\mu(A)^{2}$.

Proof. Let $D_{\delta}(x)$ be the spherical disc of radius $\delta$ with center at the point $x \in S^{2}$. By the Lebesgue theorem on density [10, theorem 2.9.11], for almost all $x$ we have $\lim _{\delta \rightarrow 0} \frac{\mu\left(A \cap D_{\delta}(x)\right)}{\mu\left(D_{\delta}(x)\right)}=\chi_{A}(x)$, where $\chi_{A}$ is the characteristic function of $A$. Therefore, $A$ contains a point $N$ with

$$
\lim _{\delta \rightarrow 0} \frac{\mu\left(A \cap D_{\delta}(N)\right)}{\mu\left(D_{\delta}(N)\right)}=1 .
$$

Choose spherical coordinates $\left(x, x_{1}\right)$ on $S^{2}$, putting the north pole at the point $N$. Norm the coordinates so that the longitude $x$ lies on the circle $S^{1}$ and the latitude $x_{1}$ lies in the segment $I=[-1,1]$. We adhere to our previous convention that $S^{1}=\mathbb{R} / \mathbb{Z}$ with the probability Lebesgue measure $\mu_{0}$. For the appropriate choice of probability measure $\mu_{1}$ on $I$, the sphere can be identified in the measure-theoretic sense with the product $S^{2}=S^{1} \times I$. For every $g \in S^{1}$ we consider symmetry $s_{g}\left(x, x_{1}\right)=\left(g-x, x_{1}\right)$, which is reflection in a plane.

Consider a symmetric set $B_{g}=A \cap s_{g}(A)$ and prove by reductio ad absurdum that for some $g \in S^{1}$ the strong inequality $\mu\left(B_{g}\right)>\mu(A)^{2}$ is true. Recall the relation (20) in the proof of Proposition 4.2. It follows that if $\mu\left(B_{g}\right) \leq \mu(A)^{2}$ for all $g$, then

$$
\int_{I} \mu_{0}\left(A_{x_{1}}\right)^{2} d \mu_{1}\left(x_{1}\right)=\left(\int_{I} \mu_{0}\left(A_{x_{1}}\right) d \mu_{1}\left(x_{1}\right)\right)^{2}=\mu(A)^{2} .
$$

The latter implies $\mu_{0}\left(A_{x_{1}}\right) \equiv \mu(A)$ almost everywhere on $I$. Therefore, for every measurable set $D \subset S^{2}$ of kind $D=S^{1} \times I_{1}$ with $I_{1} \subset I$ we have

$$
\mu(A \cap D)=\int_{I_{1}} \mu_{0}\left(A_{x_{1}}\right) d \mu_{1}\left(x_{1}\right)=\mu(A) \cdot \mu(D) .
$$


Applying this equality to $D=D_{\delta}(N)$, we have $\frac{\mu\left(A \cap D_{\delta}(N)\right)}{\mu\left(D_{\delta}(N)\right)}=\mu(A)$ for all $\delta>0$. By $(21)$ we get $\mu(A)=1$, a contradiction.

Violation of $(\mathbf{S L})$. In the rest of this section we prove the claim 3 of Theorem 4.1 showing that the disc $V^{2}$ is an exception for which Property (SL) is false.

Proposition 4.4 For any $0 \leq \alpha \leq 1 / 2$ there is a set $A \subset V^{2}$ of measure $\mu(A)=\alpha$ without symmetric subsets whose measure exceeds $\alpha^{2}$.

Proof. Instead of the disc $V^{2}$, it will be technically more convenient for us to deal with the space $V=S^{1} \times S^{1}$ supplied with the product measure $\mu_{0} \times \mu_{0}$, where $\mu_{0}$ is the Lebesgue measure on the circle $S^{1}=\mathbb{R} / \mathbb{Z}$. For this purpose we establish $f: V \rightarrow V^{2}$, a one-to-one mapping from $V$ onto the disc $V^{2}$ with the center pricked out, that will preserve measure and symmetry. We describe a point in the space $V$ by a pair of coordinates $\left(x_{1}, x_{2}\right)$ with $x_{1} \in(0,1]$ and $x_{2} \in(0,1]$, whereas for the disc $V^{2}$ we use polar coordinates $(\rho, \phi)$ with $\rho \in\left[0, \pi^{-1 / 2}\right]$ and $\phi \in(0,2 \pi]$. We set $f\left(x_{1}, x_{2}\right)=(\rho, \phi)$ iff $x_{1}=\phi /(2 \pi)$ and $x_{2}=\pi \rho^{2}$.

To explain the geometric sense of the correspondence $f$, let us identify $S^{1}$ with $(0,1]$ and regard the square $V=(0,1] \times(0,1]$ as the development of a cylinder on a plane. Then a longitudinal section of the cylinder is carried by $f$ onto a radius of the disc. A cross section is carried onto a concentric circle so that the area below the section is equal to the area within the circle. It follows that a set $X \subseteq V^{2}$ is measurable iff so is $f^{-1}(X)$, and both have the same measure.

The correspondence $f$ preserves symmetry in the following sense. For every admissible symmetry $s$ of the disc $V^{2}$ there is a transformation $s^{\prime}$ of the space $V$ such that the equality $s(X)=X$ for $X \subseteq V^{2}$ is equivalent with the equality $s^{\prime}\left(f^{-1}(X)\right)=f^{-1}(X)$. Every admissible symmetry of the disc is either a rotation around the center or a reflection in a diameter. If $s$ is the rotation by angle $2 \pi g$, then $s^{\prime}$ is definable by $s^{\prime}\left(x_{1}, x_{2}\right)=\left(g+x_{1}, x_{2}\right)$ (for the cylinder this is a rotation around its vertical axis). If $s$ is the reflection in the diameter $\phi=\pi g$, then $s^{\prime}\left(x_{1}, x_{2}\right)=\left(g-x_{1}, x_{2}\right)$ (for the cylinder this is reflection in one of its vertical planes of symmetry).

Thus, it suffices to find a set $A \subset V$ of measure $\alpha$ but without $s^{\prime}$-symmetric subsets of measure more than $\alpha^{2}$. To do so, we fix an arbitrary set $H \subset S^{1}$ of measure $\mu_{0}(H)=\alpha$ so that $H$ is completely contained in some semicircle. Then we define $A=\left\{\left(x_{1}, x_{2}\right): x_{1}+x_{2} \in H\right\}$.

It is not hard to see that $A$ has no subset symmetric with respect to any symmetry $s^{\prime}\left(x_{1}, x_{2}\right)=\left(g+x_{1}, x_{2}\right)$. Compute the measure of the maximum subset of $A$ symmetric with respect to a symmetry $s^{\prime}\left(x_{1}, x_{2}\right)=\left(g-x_{1}, x_{2}\right)$. We have

$$
\begin{array}{r}
\mu\left(A \cap s^{\prime}(A)\right)=\int_{S^{1}} \mu_{0}\left(\left\{x_{1} \in S^{1}: x_{1}+x_{2}, g-x_{1}+x_{2} \in H\right\}\right) d \mu_{0}\left(x_{2}\right) \\
\quad=\int_{S^{1}} \mu_{0}\left(H \cap\left(g+2 x_{2}-H\right)\right) d \mu_{0}\left(x_{2}\right)=\mu_{0}(H)^{2}=\alpha^{2} .
\end{array}
$$

The proposition follows. 

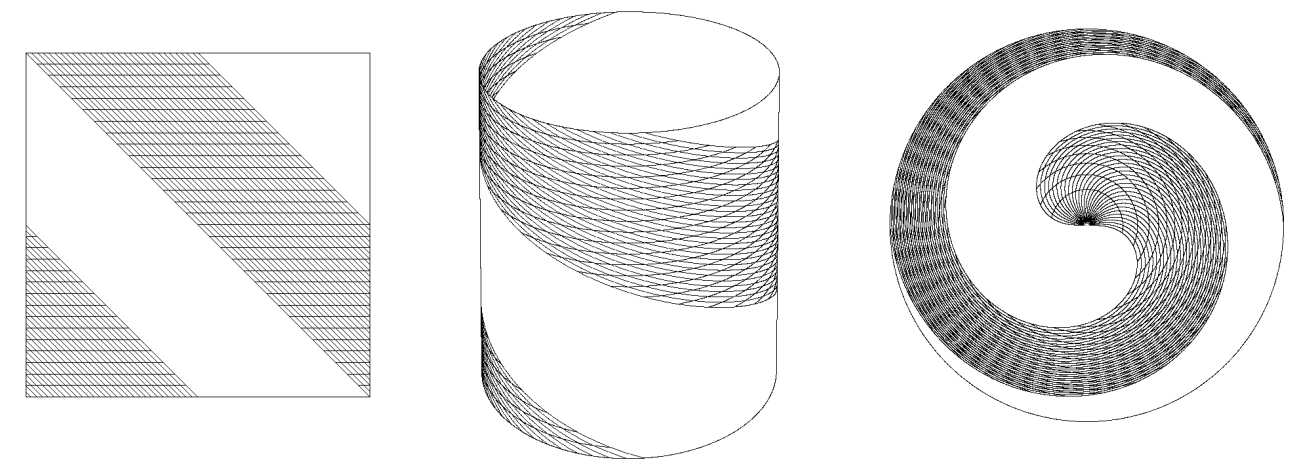

Figure 1: Construction of a bicoloring of the disc without monochromatic symmetric sets of measure more than $1 / 4$.

The above argument can be easily extended to construct an $r$-coloring of the disc without monochromatic symmetric subsets of measure more than $1 / r^{2}$. It suffices to apply the transformation $f$ to the partition $V=A_{1} \cup \ldots \cup A_{r}$, where

$$
A_{i}=\left\{\left(x_{1}, x_{2}\right): \frac{i-1}{r}<x_{1}+x_{2} \leq \frac{i}{r} \text { or } \frac{i-1}{r}<x_{1}+x_{2}-1 \leq \frac{i}{r}\right\} .
$$

For $r=2$ this coloring is shown in Figure 1.

\subsection{Figures with finite number of symmetries}

Let $G$ denote the group of all isometries of Euclidean space leaving a figure $\Omega$ invariant. Recall that for $\Omega$ we consider the family of symmetries $\mathcal{S}=G \backslash\{\mathrm{id}\}$, excluding the identity. Suppose now that $G$ is finite. In this case, which includes regular polygons, rectangles, ellipses, and their multi-dimensional analogs, the previous techniques do not apply, and we need a completely different approach.

The first thing to be understood is that the exact geometric shape of $\Omega$ is not so relevant, as the value $m s(\Omega, r)$ eventually depends only on the group $G$. For instance, $m s(\Omega, r)$ is the same for the rectangle and the ellipse (independently of whether contours or areas are meant), for the parallelopiped and the ellipsoid etc.

To be more accurate, we assume that $\Omega$ contains a measurable subset $I$ (a fundamental domain in the sense of [8]) such that all $s I$ for $s \in G$ are pairwise disjoint and $\mu\left(\bigcup_{s \in G} s I\right)=1$. In other words, $\{s I\}_{s \in G}$ is a partition of $\Omega$ into $N=|G|$ congruent pieces (points whose orbit under action of $G$ is shorter than $N$ are excluded from consideration, and their measure is assumed to be zero).

The group $G$ itself can be regarded as a space with symmetries $\sigma_{s}: G \rightarrow G$, for each $s \in G$ defined by $\sigma_{s}(g)=s g$. Denote $R=r^{N}$. Let $\phi_{1}, \ldots, \phi_{R}: G \rightarrow[r]$ be all possible $r$-colorings of $G$. Introduce notation $M_{s, i}^{j}$ to denote the cardinality of the 
THE ELECTRONIC JOURNAL OF COMBINATORICS 7 (2000), \#R52

maximum $\sigma_{s}$-symmetric subset of $G$ having color $i$ under the coloring $\phi_{j}$. Formally, $M_{s, i}^{j}=\left|\bigcap_{t=1}^{N} s^{t} \phi_{j}^{-1}(i)\right|$. In the natural way we will identify the orbit $G x=\{s(x)\}_{s \in G}$ of a point $x \in I$ with the group $G$ itself. Given an $r$-coloring $\chi$ of $\Omega$, let $I_{j}$ consist of those $x \in I$ that $\chi$ induces the coloring $\phi_{j}$ on $G x$. Set $p_{j}=\mu\left(I_{j}\right)$.

It is not hard to see that the maximum $s$-symmetric subset of $\Omega$ that receives color $i$ under the coloring $\chi$ has measure $\sum_{j=1}^{R} p_{j} M_{s, i}^{j}$. Therefore

$$
m s(\Omega, r)=\min _{\left(p_{j}\right)} \max _{\substack{s \in G \backslash\{\mathrm{id}\} \\ i \leq r}} \sum_{j=1}^{R} p_{j} M_{s, i}^{j}
$$

where the minimum is taken over all vectors $\left(p_{1}, \ldots, p_{R}\right)$ with $0 \leq p_{j} \leq 1 / N$ and $\sum_{j=1}^{R} p_{j}=1 / N$. This equality, in particular, implies that $m s(\Omega, r)$ depends only on the group $G$ of all isometries of $\Omega$.

In fact, relation (22) shows that any geometric figure with finite symmetry group $G$ is equivalent to the space $\Omega=G \times[0,1]$ with the uniform probability measure and slice-wise symmetries $\hat{\sigma}_{s}: \Omega \rightarrow \Omega$, for each $s \in G \backslash\{$ id $\}$ defined by $\hat{\sigma}_{s}(g, x)=(s g, x)$. For example, the regular $n$-gon in a plane can be identified with space $D_{2 n} \times[0,1]$, where $D_{2 n}$ is the dihedral group; and the $k$-dimensional parallelopiped (as well as ellipsoid) can be identified with space $\mathbb{Z}_{2}^{k} \times[0,1]$.

\section{Theorem 4.5}

(1) Let $p$ be the smallest prime divisor of $n$. Then for the regular $n$-gon $\Gamma_{n}$ we have

$$
m s\left(\Gamma_{n}, r\right)= \begin{cases}\frac{p-1}{p^{2}+2 p-2} & \text { if } r=2, \\ \frac{1}{3 p^{2}+6} & \text { if } r=3 \\ 0 & \text { if } r \geq 4\end{cases}
$$

(2) For the $k$-dimensional parallelopiped $\Pi_{k}$ we have

$$
m s\left(\Pi_{k}, r\right)=\frac{2^{k}-r}{r^{2}\left(2^{k}-1\right)}
$$

whenever $r=2^{l}$ for some $0 \leq l<k$.

The proof of Theorem 4.5 has not been published yet and will appear elsewhere.

\section{$\S 5 \quad$ Extremal colorings}

Given an $r$-coloring $\chi$ of a space $\Omega$ with symmetry, let $m s(\Omega, r ; \chi)$ denote the supremum of $\mu(A)$ over all $\chi$-monochromatic symmetric sets $A \subseteq \Omega$. We call $\chi$ 
extremal if $m s(\Omega, r ; \chi)=m s(\Omega, r)$. Similarly, a blurred $r$-coloring $\beta$ is extremal if bms $(\Omega, r ; \beta)=b m s(\Omega, r)$ (see Definition 2.4).

Obviously, no extremal colorings exist whenever both of Properties (U) and (SL) are met, in particular, for a wide variety of compact Abelian groups (see Section 3.3), for spheres of all dimensions, and for balls starting from dimension 3 (see Theorem 4.1). By Theorem 4.1 (3), an extremal coloring does exist for the 2-dimensional disc. We do not know what is the case for the space $\Omega=[0,1]$.

Whenever $\operatorname{bms}(\Omega, r)=1 / r^{2}$, there exists the obvious extremal blurred coloring $\beta=$ $\left\{\beta_{i}\right\}_{i=1}^{r}$ with each $\beta_{i}=1 / r$ everywhere on $\Omega$. In particular, this is the case for the aforementioned spaces without extremal "distinct" colorings. For the segment $[0,1]$ the answer is not so obvious. The proof of the following result can be found in $[5$, theorem $7.2]$.

Theorem 5.1 There exists an extremal blurred $r$-coloring of $[0,1]$.

One could expect that extremal colorings, if exist, have some regular properties. Observe that color classes of the extremal coloring of the disc constructed in Section 4.1 are congruent (see Figure 1). In particular, if there is a symmetric set of one color with measure $\alpha$, then there must be a symmetric set of any other color with the same measure. The latter property is actually fulfilled for all extremal colorings of the disc. This can be inferred from relation (20) in the proof of Proposition 4.2. It turns out that an analog of this property for blurred colorings is true for all spaces with symmetry. More precisely, if $\beta=\left\{\beta_{i}\right\}_{i=1}^{r}$ is an extremal blurred $r$-coloring of a space $\Omega$, i.e. $\operatorname{bms}(\Omega, r)=\left\|\beta_{i} \star \beta_{i}\right\|$ for some $i \leq r$, then the same equality holds true for all $i \leq r$.

\section{$\S 6$ Infinitary issues}

In this section we reconsider our original problem from another perspective. Modifying the setting of Definition 1.6 in a quantitative aspect, we become concerned with the cardinality of a monochromatic symmetric subset rather than with its measure. Given a space $\Omega$ with symmetry and a cardinal number $\kappa$, the proper question to ask now is what minimum (cardinal) number $r$ of colors suffices to color $\Omega$ so that there were no monochromatic symmetric subsets of cardinality $\kappa$.

As first example, consider an Abelian group $G$ with symmetries $s_{g}(x)=g-x$. Define $\nu(G)$ to be the minimal $r$ such that there exists an $r$-coloring of $G$ without infinite monochromatic symmetric subsets. The following result is proved in [3].

Theorem $6.1 \nu\left(\mathbb{Z}^{k}\right)=k+1$.

Proof. To show that $\nu\left(\mathbb{Z}^{k}\right) \leq k+1$, define a $(k+1)$-coloring of $\mathbb{Z}^{k}$ with color classes $A_{1}, \ldots, A_{k+1}$ as follows. Consider a $k$-dimensional simplex $S$ (a segment in $\mathbb{R}$, a triangle in $\mathbb{R}^{2}$, a tetrahedron in $\mathbb{R}^{3}$ and so on). Fix a point $p$ inside $S$. For a point $z \in \mathbb{R}^{k}$, 
let $R(z)$ be the ray extending from $p$ and passing through $z$. Let $A_{i}$ consist of those lattice points $z$ that $R(z)$ intersects $i$-th face of $S$. Clearly, no $A_{i}$ contains an infinite symmetric subset.

Now we need to prove that in any $k$-coloring of $\mathbb{Z}^{k}$ one can find an infinite monochromatic symmetric set. The one-dimensional case is trivial, and the two-dimensional case is still not so hard. We outline the proof for the first non-trivial case of $k=3$ that can be easily extended to higher dimensions.

Suppose the contrary and consider a 3 -coloring of $\mathbb{Z}^{3}$ without infinite monochromatic symmetric sets. Let $C=\{-1,0,1\}^{3}$ be a discrete cube and $K=[-m, m]^{3}$ a continuous cube in $\mathbb{R}^{3}$. It follows from our assumption that if $m$ is large enough, then the boundary $\partial K$ of $K$ contains no two lattice points of the same color and symmetric with respect to a center in $C$. Fix such a cube $K$ for some even $m$. Triangulate $\partial K$ into isosceles rightangled triangles with vertices in all those lattice points of $\partial K$ whose three coordinates are even. For convenience we choose this triangulation symmetric with respect to the origin $(0,0,0)$.

Fix now a triangle $T$ in $\mathbb{R}^{2}$ and assign each of three colors to one of the vertices of $T$. Define a mapping $h: \partial K \rightarrow T$ by the following two conditions.

(1) $h$ takes each lattice point of $\partial K$ with all three coordinates even (i.e. each vertex of the triangulation) into the vertex of $T$ with the same color.

(2) The mapping $h$ is linear on each element of the triangulation. In other words, for every triangle $T^{\prime}$ in the triangulation of $\partial K$, there is a linear transformation from $\mathbb{R}^{3}$ to $\mathbb{R}^{2}$ that induces $h: T^{\prime} \rightarrow T$.

Clearly, $h$ is uniquely determined by these two conditions and is continuous. Apply to $h$ the Borsuk-Ulam antipodality theorem (see e.g. [7, theorem 13.6]). It follows that there exists a pair $\{x,-x\}$ of antipodal points on $\partial K$ with $h(x)=h(-x)$. Let $a, b, c \in \partial K$ be vertices of the triangle containing the point $x$ (if $x$ lies on the border between two or more triangles, we merely choose one of them). The triangle with vertices $-a,-b,-c$ contains the point $-x$. By the linearity of $h$, images $h(x)$ and $h(-x)$ belong to the convex hulls of sets $\{h(a), h(b), h(c)\}$ and $\{h(-a), h(-b), h(-c)\}$ respectively. Consequently, the two convex hulls have nonempty intersection.

On the other hand, any point in $\{a, b, c\}$ is symmetric to any point in $\{-a,-b,-c\}$ with respect to a center in $C$. By our assumption, colors of $\{a, b, c\}$ and colors of $\{-a,-b,-c\}$ do not intersect and, hence, $\{h(a), h(b), h(c)\}$ and $\{h(-a), h(-b), h(-c)\}$ are disjoint sets of vertices of the triangle $T$. Therefore, convex hulls of these two sets are disjoint too, a contradiction.

In [3] the cardinal $\nu(G)$ is computed for any Abelian group $G$. Other relevant questions are discussed in $[1,2]$. The following statement has been recently proven by Igor Protasov and the first author [4] based on a version of the Erdös-Rado partition theorem. 
THE ELECTRONIC JOURNAL OF COMBINATORICS 7 (2000), \#R52

Theorem 6.2 Assume that the generalized continuum hypothesis is true. Let $G$ be an infinite Abelian group whose order exceeds a cardinal number $\kappa$. Then, for any coloring of $G$ in finite number $r$ of colors, $G$ contains a monochromatic symmetric subset of cardinality at least $\kappa$.

According to an earlier result of Protasov [20], for $r \leq 3$ the theorem can be proved without the generalized continuum hypothesis. If $\kappa$ is equal to the order of $G$, the statement is not true.

\section{$\S 7$ Open problems}

1. Improve our bounds on $m s([0,1], 2)$. In particular, a better upper bound can be attained just at cost of more calculation, namely, by more careful estimating particular values $\operatorname{bms}([n], 2)$ for $n \geq 4$.

2. Does there exist an extremal blurred bicoloring $\left\{\beta_{1}, \beta_{2}\right\}$ of $[n]$ such that $\beta_{1}(x)=$ $\beta_{2}(n+1-x)$ for all $x \in[n]$ ? If so, this would facilitate computation of particular values bms $([n], 2)$.

3. Improve our bounds on the constant $c$ in Theorem 2.1 (2). In particular, can it be separated from $1 / 2$ ?

4. How fast does the sequence $m s([n], r)$ converge? In particular, is the bound $|m s([n], r)-m s([0,1], r)|=O\left(1 / n^{\alpha}\right)$ true for a positive $\alpha$ ? How faster is convergence of $b m s([n], r)$ ?

5. Does there exist an extremal coloring of the segment $[0,1]$ ? In particular, is the equality $m s([0,1], r)=m s([n], r)$ possible for some $n$ ? Recall that the value $m s([0,1], r)=b m s([0,1], r)$ is achievable by a measurable blurred coloring of the segment. Is it achievable by a piecewise-continuous blurred coloring?

6. For a space $\Omega$ with symmetry and a real $\sigma$ define

$$
d m s(\Omega, \sigma)=\inf \{m s(A): A \subseteq \Omega, \mu(A) \geq \sigma\} .
$$

Clearly, $m s(\Omega, r) \geq d m s(\Omega, 1 / r)$. If $\Omega$ has Property $(\mathrm{L})$, then $d m s(\Omega, \sigma) \geq \sigma^{2}$. Thus, whenever $\Omega$ has both of Properties $(\mathrm{L})$ and $(\mathrm{U})$, we have the equality $m s(\Omega, r)=$ $d m s(\Omega, 1 / r)$. Is this equality true for $\Omega=[0,1]$ ?

7. (P. Erdős, A. Sárközy, V. T. Sós [9]) Given an $r$-coloring $\chi:[n] \rightarrow[r]$, let $S(g)$ denote the sum of the cardinalities of all monochromatic sets symmetric with respect to $\frac{1}{2} g$, i.e., $S(g)=\sum_{i=1}^{r}\left|\chi^{-1}(i) \cap\left(g-\chi^{-1}(i)\right)\right|$. Does there exist a positive constant $c(r)$ such that, for every $r$-coloring of $[n]$, the bound $S(g) \geq c(r) g$ is true for almost all even integers $g$ not exceeding $n$ ? A statement of such a kind is proven in [9] with the bound $S(g) \geq 2$. This cannot be extended to odd integers $g$, because if $\chi$ colors all even numbers in $[n]$ and all odd numbers in $[n]$ in two different colors, then $S(g)=0$ for every odd $g$.

8. The notion of symmetry can be extended also over any non-Abelian group $G$. Namely, the symmetry $s_{g}: G \rightarrow G$ with respect to an element $g$ can be defined by 
$s_{g}(x)=g x^{-1} g$. As every compact topological group has the unique probability Haar measure, for any group $G$ of this class it makes sense to consider the value $m s(G, r)$. For instance, consider the group $S O(3)$ of orientation-preserving rotations of the 3dimensional space. What is $m s(S O(3), 2)$ equal to? We can suggest the bounds $1 / 16 \leq$ $m s(S O(3), 2) \leq 1 / 4$.

9. Let $\mathcal{P}$ be the family of all orientation-preserving involutive symmetries of the sphere $S^{2}$, i.e. all its axial symmetries. What is $m s\left(S^{2}, \mathcal{P}, r\right)$ equal to? How far from the true value is the upper bound $1 / r^{2}$ ? Notice that $\mathcal{P}$ contains the group $\mathbb{Z}_{2}^{2}$, which is generated by rotations of $180^{\circ}$ around three pairwise perpendicular axes. It follows that $m s\left(S^{2}, \mathcal{P}, r\right) \geq m s\left(\mathbb{Z}_{2}^{2} \times[0,1], r\right)$, where the space $\mathbb{Z}_{2}^{2} \times[0,1]$ is considered with slice-wise symmetries as in Section 4.2. Applying Theorem 4.5 (2) for $k=2$, we obtain the lower bound $m s\left(S^{2}, \mathcal{P}, 2\right) \geq 1 / 6$.

10. Compute $m s(\Omega, r)$ for convex regular polyhedra in $\mathbb{R}^{k}$. This research program is of great interest even if restricted to particular directions. For example, it would be desirable to know $m s\left(\Delta_{k}, r\right)$ for the regular $k$-dimensional simplex $\Delta_{k}$. Equivalently one can consider the space $S_{k} \times[0,1]$, where $S_{k}$ is the symmetric group of degree $k$. Another interesting direction is to compute $m s(\Omega, r)$ for all five Platonic solids in $\mathbb{R}^{3}$.

11. (R. I. Grigorchuk) Let $F_{2}$ be a free group of rank 2. Compute $\nu\left(F_{2}\right)$ for the symmetries as in Problem 8.

12. Call a subset $E$ of an Abelian group $G$ essential if for every coloring of $G$ in less than $\nu(G)$ colors the group $G$ contains an infinite subset $A$ symmetric with respect to a point $g$ in $E$ (i.e. $A=2 g-A$ ). For example, the proof of Theorem 6.1 shows that the 27 -element cube $\{-1,0,1\}^{3}$ is an essential set in $\mathbb{Z}^{3}$. Let $\rho(G)$ denote the minimum cardinality of an essential set in $G$. The problem is to compute or to estimate $\rho\left(\mathbb{Z}^{k}\right)$. We know the bounds $k(k+1) / 2 \leq \rho\left(\mathbb{Z}^{k}\right)<2^{k}$, where the lower bound is the precise value for $k=1,2,3$ (see [2]).

13. Is Theorem 6.2 provable in $\mathrm{ZFC}$ ? In particular, can one prove without additional set-theoretic assumptions that for every 4-coloring of $\mathbb{R}$ and for every cardinal number $\kappa<\mathfrak{c}$ there exists a monochromatic symmetric set $A \subset \mathbb{R}$ of cardinality at least $\kappa$ ?

14. Let $M(n)$ be the maximum $M$ such that every sequence $v_{0}, v_{1}, \ldots, v_{n}$ of points in $\mathbb{Z}^{2}$ with each difference $v_{i+1}-v_{i}$ being either $(0,1)$ or $(1,0)$ contains an $M$-point centrally symmetric subset. What is asymptotics of $M(n)$ ? We only know that $2 \log n-O(1) \leq$ $M(n) \leq(7+o(1)) \sqrt{n}($ see $[22])$.

\section{Acknowledgments}

We appreciate the contribution of Igor Protasov, whose results and questions gave us an inspiration for this research. The second author is greatly indebted to the Department of Information Systems at Vienna University of Technology for hospitality during his work on this survey. 
THE ELECTRONIC JOURNAL OF COMBINATORICS 7 (2000), \#R52

\section{References}

[1] T. Banakh. On asymmetric colorings of Abelian groups. In: Paul Erdös and his Mathematics. Research Communications of the conference held in the memory of Paul Erdös, July 4-11, 1999, Budapest, Janos Bolyai Mathematical Society, 30-32.

[2] T. Banakh. On a cardinal group invariant related to partitions of Abelian groups (in Russian). Mat. Zametki, 64(3):341-350, 1998. English translation in Math. Notes, 64(3):295-302, 1998.

[3] T. Banakh and I. Protasov. Asymmetric partitions of Abelian groups (in Russian). Mat. Zametki, 66(1):10-19, 1999. English translation in Math. Notes, 66(1):8-15, 1999.

[4] T. Banakh and I. Protasov. Symmetry and colorings: some results and open problems. Voprosy Algebry, 2000, to apppear.

[5] T. Banakh, Ya. Vorobets, and O. Verbitsky. Ramsey-type problems for spaces with symmetry (in Russian). Izvestiya Ross. Akad. Nauk, Ser. Mat. (Russian Academy of Sciences. Izvestiya. Mathematics), 2000, to appear.

[6] E. R. Berlekamp. A construction of partitions which avoid long arithmetic progressions. Canad. Math. Bull., 11:409-414, 1968.

[7] A. Björner. Topological methods. In Handbook of Combinatorics, chapter 34, pages 1819-1872. Elsevier Publ., 1995.

[8] H. S. M. Coxeter. Introduction to geometry. 2nd ed., New York etc.: John Wiley and Sons, 1969.

[9] P. Erdős, A. Sárközy, V. T. Sós. On a conjecture of Roth and some related problems I. In Irregularities of partitions, Pap. Meet., Fertod/Hung. 1986. Algorithms and Combinatorics 8, pages 47-59, 1989.

[10] H. Federer. Geometric measure theory. Berlin-Heidelberg-New York: SpringerVerlag, 1969.

[11] R. L. Graham, B. L. Rothschild, and J. H. Spencer. Ramsey theory. 2nd ed., WileyInterscience Series in Discrete Mathematics and Optimization. New York etc.: John Wiley \& Sons, 1990.

[12] F. Krückeberg. $B_{2}$-Folgen und verwandte Zahlenfolgen. J. Reine Angew. Math., 206:53-60, 1961.

[13] L. H. Loomis. An introduction to abstract harmonic analysis. Toronto-New YorkLondon: D. Van Nostrand Company, 1953. 
THE ELECTRONIC JOURNAL OF COMBINATORICS 7 (2000), \#R52

[14] S. A. Morris. Pontryagin duality and the structure of locally compact Abelian groups. London Mathematical Society Lecture Note Series 29. Cambridge University Press, 1977.

[15] J. Nešetřil. Ramsey theory. In Handbook of Combinatorics, chapter 25, pages 1331-1403. Elsevier Publ., 1995.

[16] J.-L. Nicolas and G. Robin. Majorations explicites pour le nombre de diviseurs de N. Canad. Math. Bull., 26:485-492, 1983.

[17] C. Pomerance and A. Sárközy. Combinatorial number theory. In Handbook of Combinatorics, chapter 20, pages 967-1018. Elsevier Publ., 1995.

[18] K. Prachar. On integers having many representations as a sum of two primes. J. London Math. Soc., 29:347-350, 1954.

[19] I. Protasov. Asymmetrically decomposable Abelian groups (in Russian). Mat. Zametki, 59(3):468-471, 1996. English translation in Math. Notes, 59(3):336-338, 1996.

[20] I. Protasov. Monochromatic symmetric subsets in the colorings of Abelian groups. Dopovidi Natsionalnö̈ Akademï Nauk Ukraïny, 11:54-57, 1999.

[21] L. G. Schnirelmann. Über additive Eigenschaften von Zahlen. Math. Ann., 107:649690, 1933.

[22] O. Verbitsky. Symmetric subsets of lattice paths. INTEGERS: an Electronic Journal of Combinatorial Number Theory, Vol. 0, A05, 16 pages, 2000.

[23] H. Weyl. Symmetry. Reprint of the 1952 original. Princeton Science Library. Princeton, NJ: Princeton University Press, 1989. 